\title{
Patient and Caregiver Insights into the Disease Burden of Myelodysplastic Syndrome
}

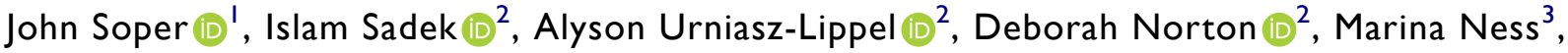 \\ Ruben Mesa $\mathbb{1 D}^{4}$
}

'Bioaeronautical Research Laboratory, Civil Aerospace Medical Research Institute, Oklahoma City, OK, USA; ${ }^{2}$ Novartis Pharmaceuticals Corporation, East Hanover, NJ, USA; ${ }^{3}$ Inspire, Arlington, VA, USA; ${ }^{4}$ Mays Cancer Center at UT Health San Antonio MD Anderson, San Antonio, TX, USA

Correspondence: Ruben Mesa, Mays Cancer Center at UT Health San Antonio MD Anderson, 7979 Wurzbach Road, San Antonio, TX, 78229, USA, Tel +I 210-450-1724, Fax + 210-450-II00, Email mesar@uthscsa.edu

\begin{abstract}
A diagnosis of myelodysplastic syndrome (MDS) is typically unexpected and can be difficult for patients to grasp. Not only is MDS a complicated disease to understand, which can contribute to stress and anxiety, but it also has an uncertain prognosis, which can be emotionally paralyzing. Not surprisingly, emotional distress and the symptom burden of MDS, including extreme fatigue due to cytopenias, negatively impact a patient's quality of life (QOL). Studies have shown that patient-centered care-including greater physician understanding of the disease burden their patients experience, discussing and establishing agreed-on treatment goals, and including patients in the decision-making process about their care-may help improve patient QOL. To better understand patient and caregiver experiences with MDS and how the disease impacts QOL, a small survey was conducted of patients with MDS or leukemia and their caregivers on an online health network. Among the 30 respondents who completed the survey, four had MDS and one was a caregiver for a patient with MDS. Here we focus on the five MDS respondents and contextualize the findings with personal experiences from a patient and physician perspective. The patient perspective was provided by John Soper, PhD, DABCC, who was diagnosed with MDS in 2019. Dr Soper is a retired board-certified clinical chemist and a member of the MDS Foundation. The physician perspective was provided by Dr Ruben Mesa, Executive Director of the Mays Cancer Center at UT Health San Antonio MD Anderson. The survey responses and the accompanying patient and physician perspectives highlight the importance of open communication between patients and their healthcare provider to better serve those with MDS and improve their QOL.
\end{abstract}

Keywords: quality of life, survey, online health network, personal experiences

\section{Background}

Patients with myelodysplastic syndrome (MDS) have a substantially reduced quality of life (QOL) because of emotional distress and symptom burden. ${ }^{1,2}$ After diagnosis, the disease typically becomes a dominating factor in patients' lives, impacting nearly every facet. ${ }^{3}$ The symptoms associated with cytopenias are often debilitating, and day-to-day living is upended by the hassle of medical care.

In the United States, healthcare providers and patients agree that compassionate, patient-centered care is important, but poor communication remains prevalent. ${ }^{4,5}$ Perception of symptom burden, QOL, and disease characteristics between patients with MDS and their physicians is often discordant, which may result in poor patient satisfaction, erode patient trust, compromise treatment decision-making, and negatively impact patient outcomes. ${ }^{6,7}$ For instance, while assessment of QOL is associated with improved survival in patients with cancer, many oncologists do not administer QOL assessments or discuss QOL with their patients. ${ }^{89}$ Patient-centered communication may help resolve the discordance in physician vs patient understanding of MDS disease and the treatment journey, ${ }^{10}$ underscoring the need for greater physician insight into the patient experience.

In recognition of the need to increase physician insight into a patient's treatment and disease journey, a survey was conducted of patients with MDS or leukemia and their caregivers on an online health network comprising more than 2 
million members and 250 online support communities. The purpose of the survey was to better understand patient and caregiver experiences in relation to the patient's disease and treatment, and the impact these facets have on the patient's QOL. Among the 30 qualified respondents who completed the survey, four had MDS and one was a caregiver for a patient with MDS.

Here, we discuss the results of this survey, focusing on the five MDS respondents. The findings are contextualized with personal experiences from both a patient and physician perspective to better elucidate the importance of more open healthcare provider and patient dialogues throughout the MDS disease and treatment journey. The patient perspective is provided by John Soper, PhD, DABCC, who was diagnosed with MDS in 2019. Dr Soper is a retired board-certified clinical chemist and a member of the MDS Foundation. The physician perspective is provided by Dr Ruben Mesa, Executive Director of the Mays Cancer Center at UT Health San Antonio MD Anderson. Dr Mesa has more than 20 years of experience in clinical research of myeloproliferative neoplasms and MDS, with leadership in more than 100 clinical trials for these disorders.

\section{Diagnosis}

MDS is not an easy or straightforward diagnosis due to the pathologic and clinical heterogeneity of the disease. ${ }^{11,12}$ It can also be difficult to distinguish from other cytopenias, including iron-deficiency anemia. ${ }^{12}$ Patients with suspected MDS will undergo a number of tests including complete blood counts, marrow morphology, cytogenetics, and evaluation for other causes of cytopenia. ${ }^{12}$ Thus, a confirmative diagnosis of MDS can take weeks to months, which can cause distress and uncertainty for many patients.

A diagnosis of MDS is often an emotionally devastating experience for patients, frequently leading to depression and anxiety. ${ }^{3}$ In the survey, two of five respondents were unaware or unsure of the specific type of MDS they or their loved one was diagnosed with; this may be reflective of gaps in patient-centered communication. Distress in MDS is associated with patient health literacy and physician communication more so than MDS disease characteristics, underscoring the need to improve patient-centered communication. ${ }^{13}$ The importance of mental health distress screening in the oncology setting has been well established, ${ }^{14}$ but assessment and management of mental health in patients with cancer remain suboptimal. ${ }^{15}$

\section{Patient Perspective on Diagnosis: Dr Soper}

My journey with MDS began following a quintuple cardiac bypass 5 years ago. As part of the course of care, my cardiologist discovered that I had macrocytic anemia. After an oncologist evaluated me, I was monitored for 3 years until I was definitively diagnosed, but I had known for some time that I likely had MDS. For many other patients, however, the diagnosis comes out of the blue. My oncologist was proactive regarding mental health and ensured that I would receive treatment for depression when needed. I was not crippled with depression and anxiety per se, but it is a fact that this disease is mentally distressing to patients, and I am thankful that my oncologist had this awareness regarding mental health. In speaking with other patients with MDS, I have found that mental health issues are unfortunately not openly discussed.

Sharing my journey with other patients and learning about their experiences has been a critical part of the process for me. This is not something that any of my doctors suggested, but it has come about organically throughout the course of my disease. As a retired clinical chemist, I have always looked at this disease through the lens of a scientist. I have been proactive about following my own clinical laboratory data trends and learning more about the disease, but I know that it is common to be terrified by the information and to prefer to not know. Moreover, many other patients do not have the background in science that I do, so even those who want to know more can often struggle. This, to me, underscores the importance of having excellent physician-patient communication.

\section{Physician Perspective on Diagnosis: Dr Mesa}

At diagnosis, most patients have never even heard of MDS, and this lack of familiarity contributes to anxiety, distress, and difficulty in understanding the disease. Because diagnosis is often delayed, patients most likely have already suffered from longstanding cytopenias with symptoms such as fatigue, shortness of breath, infections, and bleeding. Immediately 
the patient's life is changed: the inconveniences of physician visits, the symptoms of cytopenias, the toxicities of treatments, and the worry of the unknown become constant realities. For the entirety of the disease journey, physician-patient communication can help the patient better understand what is happening and feel more in control. Likewise, physicians who become more in tune with their patient's experience can better respond to evolving needs.

\section{Treatment Decisions}

Patient-centered communication can empower patients to better understand their disease and take a greater role in their relationship with their oncologist. ${ }^{16}$ Despite the value to patients, direct conversations about treatment goals often occur late in the treatment journey; a recent study found that, among patients with terminal cancer who discussed treatment goals with their healthcare providers, discussions began on average 1 month prior to the patient's death and occurred primarily in acute care settings. ${ }^{17}$ Less than $20 \%$ of healthcare practitioners agreed with patients regarding the most important goals of treatment. ${ }^{18}$ For patients, the most important goal was "slow/delay progress of conditions" whereas for healthcare providers, "symptom improvement" and "prevention of vascular/thrombotic events" were regarded as the primary goals. Proactively ascertaining patient treatment preferences assists with treatment adherence and decisionmaking. ${ }^{6}$

The survey results showed that the respondents' conception of MDS differed, suggesting disparities in physician communication about the disease. Two respondents did not describe their disease as a cancer or malignancy. One respondent believed that MDS is "more aggressive than leukemia" and that outcomes in patients with MDS are "less positive" than those with leukemia. This lack of understanding about their disease may impede a patient's ability to participate in the treatment decision-making process. Notably, all respondents indicated that they wanted to be involved in treatment selection and reported that they had asked their providers to explain all treatment options before initiating a therapy. Respondents all sought information regarding MDS from sources such as medical research organizations, online search engines, peer-reviewed journals, patient organizations, and disease specialists. Two respondents stated that they were "always looking for information," which may indicate they are not getting the information they desire or need from their physicians. Three of the respondents reported that information was difficult to understand. Respondents also wanted to see more patient-reported information.

\section{Patient Perspective on Treatment Decisions: Dr Soper}

Communication with my physicians has been a key part of my treatment journey. Early on, I discussed treatment goals, and when beginning new therapies, important factors like insurance coverage were considered. I was also enrolled in a clinical trial and established regular communication with the lead investigator. Overall, my healthcare providers are responsive to me and take my treatment suggestions seriously. This is profoundly important to me and leads to consensus-based treatment decisions that all of us are comfortable with.

\section{Physician Perspective on Treatment Decisions: Dr Mesa}

A key unmet need in communication is that many patients do not accurately understand what MDS is. It is a challenging disease to understand for someone without advanced health literacy; accurate discussions around diagnosis, prognosis, treatment planning, and goals are all critical. It is important for physicians to carefully consider how to frame the disease in the patient's mind as well as what successful treatment looks like, and we must forge a shared understanding of all aspects of the treatment journey. Patients are often fundamentally misaligned regarding the nature of their treatments, and may believe that erythropoietin, for example, is curative.

\section{Treatment Journey and Challenges}

With the profound clinical heterogeneity of MDS, ${ }^{19}$ the treatment journey of patients with MDS is not a straightforward path; symptom burden, changing treatments, enrollment in clinical trials, and other factors can change the patient's course. Conversations with patients about fatigue, social functioning, and other challenges they encounter should be continual throughout the treatment journey to help physicians better understand the implications treatment decisions may have on a patient's life. ${ }^{20}$ 
Four of five survey respondents cited fatigue as the most challenging symptom to deal with; other reported symptoms included bruising, bleeding, anemia, nausea, changes in senses, rashes, diarrhea, mouth ulcers, and infections. Three respondents reported visiting infusion centers to receive their treatment multiple times per month; based on individual circumstances, this may represent a disruption to a patient's routine or be challenging to manage. For instance, patients without reliable transportation may find it difficult to attend their appointments. Additionally, three respondents noted that they had less time and attention to give to family and friends due to their treatments.

\section{Patient Perspective on Treatment Journey and Challenges: Dr Soper}

Having to go to a hospital and receive treatments regularly is something that, since my diagnosis, much of my life has centered around, and this in and of itself is difficult to deal with. The single biggest challenge of my treatment journey, however, has been fatigue. I am always tired and in a constant mental fog. Going to the hospital and receiving my treatment takes up half a day, and after I finish, I am exhausted. At some point, I became transfusion dependent. Initially, transfusions improved my fatigue, but because getting the transfusion now requires an entire day, the process leaves me drained.

\section{Physician Perspective on Treatment Journey and Challenges: Dr Mesa}

Red blood cell transfusions have an enormous impact on the patient, but key details, like the duration of transfusions and how they will affect the patient, are often missing from clinical conversations. First of all, patients are confronted with adverse effects of transfusion, such as fatigue, dizziness, and pain. As Dr Soper has mentioned, there's also an enormous hassle with transfusion - it tethers the patient to the medical center and makes it hard to plan trips or manage other aspects of life. Patients who are dependent on transfusions are kept at the minimum hemoglobin level to minimize transfusion toxicities, but the trade-off is that they spend most of their time with suboptimal hemoglobin.

\section{Symptom Burden, Disease Progression, and Other Treatment Setbacks: “The Drugs Work Until They Don't"}

Disease progression and treatment failure are often emotionally devastating to patients with cancer. ${ }^{21,22}$ In MDS, treatment setbacks may occur due to evolving symptom burden, adverse effects of treatments, and feasibility or availability of treatments.

Three of five respondents reported that their disease has had an effect on their employment, including having to cut back on hours from work, having to decline work opportunities, being passed over for career advancement, and forced retirement. Fatigue, nausea, and vomiting were noted as the most difficult treatment adverse effects to manage. The caregiver respondent reported that their patient's challenges with pneumonia during treatment represented the scariest moments of the disease journey.

\section{Patient Perspective on Disease Progression and Other Treatment Setbacks: Dr. Soper}

I do not know how much time I have left. Two years? Five? The uncertainty of this disease is frustrating, but there's nothing anyone can do about that, no matter how closely my blasts are monitored. As one of my friends with MDS put it, "the drugs work until they don't"- and one day my treatment will stop working, too. But it's important to not let the specter of the unknown control my life, so I will rejoice in each new day I have.

\section{Physician Perspective on Disease Progression and Other Treatment Setbacks: Dr Mesa} Oftentimes there's not a shared understanding between the patient and the care team as to what "progression" means. Is it increased blasts? Continued need for transfusions? Hospitalization? The endgame with many therapies is not well discussed with patients, which is compounded by the fact that many physicians do not believe they have good backup options. The consequence of this is, with patients not having a clear idea of what to expect, end-of-life care planning becomes more difficult. Overall, physicians need to address gaps in communication regarding progression, prognosis, and limitations of care. 


\section{Clinical Trials}

Clinical trial enrollment is a current standard of care for the treatment of patients with MDS. ${ }^{12}$ Numerous opportunities exist in the clinical trial setting to improve patient-centered communication. Physicians should advocate for clinical trial participation, as it is a standard of care, but beyond that, they should be ready to discuss the details of clinical trial involvement with their patients. This conversation can include factors such as what to expect, the impact on current care regimens, what the treatments are, the trial design, reasonable expectations for outcomes, how the trial will contribute to the development of MDS treatments, and what to expect afterward (follow-up, further trials, etc).

Furthermore, investigating physicians should strive for patient-enhanced communication with their study participants, as they would in the nontrial clinical setting. Clinical trials should more cohesively capture QOL endpoints, which will further enhance physician insight into the patient experience. While some oncology trials do assess QOL, these assessments are often made only during the intervention phases; less than 4\% investigate QOL until patient death, and those that do demonstrate worse QOL outcomes for intervention arms vs control arms. ${ }^{23}$ Extended assessment provides crucial insight into the evolving nature of the disease and longer-term effects of treatment.

\section{Patient Perspective on Clinical Trials: Dr Soper}

I am extremely fortunate that my physicians facilitated my enrollment in a clinical trial. Without being in a clinical trial, I would not have access to the drugs I am now receiving. I do not know what I'd be doing without a clinical trial. I do know that it has enhanced my care greatly and that I am proud to contribute to the research that will improve the treatment landscape and hopefully lead to curative options. I am being managed by one of the world's best groups for MDS. However, living far from the clinical trial site is challenging. I know patients who have sold their homes and moved to be closer to their clinical trial location, which is yet another point showing how every aspect of this disease monopolizes a patient's life. I also know other patients who never had the opportunity to enroll in clinical trials due to their location, or because their physicians never broached the topic. Reflecting on the superb care I have received due to being in a clinical trial, I think every patient with MDS should have the same opportunity to enroll.

\section{Physician Perspective on Clinical Trials: Dr Mesa}

Without a broadly applicable therapy that has curative potential or significantly impacts the disease course, clinical trials will remain essential for MDS. Trials for previously untreated patients should be structured so that the intervention is as least as effective as the standard of care. Additionally, more clinical trials are needed that allow patients for whom the standard of care has failed. As Dr Soper stated, however, clinical trials can be burdensome to patients if they are not near a trial site; I am hopeful that lessons we have learned from adapting to the COVID-19 pandemic may help proliferate clinical trials and ease patient burden. For instance, we can use telemedicine to potentially accrue and monitor patients remotely. Making the clinical trial process smooth for patients is important because they may participate for a long time.

\section{Conclusion}

At every step of the MDS disease journey, physicians have an opportunity to ease anxiety and distress for their patients (Figure 1). Starting with the diagnosis, it is important that patients receive clear and understandable information about their disease, including prognosis and current limitations of care. Patients who have a good understanding of their disease will be better equipped to participate in treatment decisions, which can be empowering for them and may offer some feeling of control over a disease that is not curable. Physicians should seek opportunities and encourage their patients to participate in clinical trials, which can ease the financial burden of treatment for some and provide the best available options for care. Greater physician insight into the experiences of patients with MDS is a current unmet need in the management of the disease. It is important for physicians to listen to individual experiences and the challenges their patients are facing during the course of the disease, and to have regular communication about their mental health, QOL, symptoms, challenges of treatment, and expectations as the disease changes over time. Patient-physician communication impacts every aspect of the disease and improving this dynamic will ultimately enhance patient outcomes. 


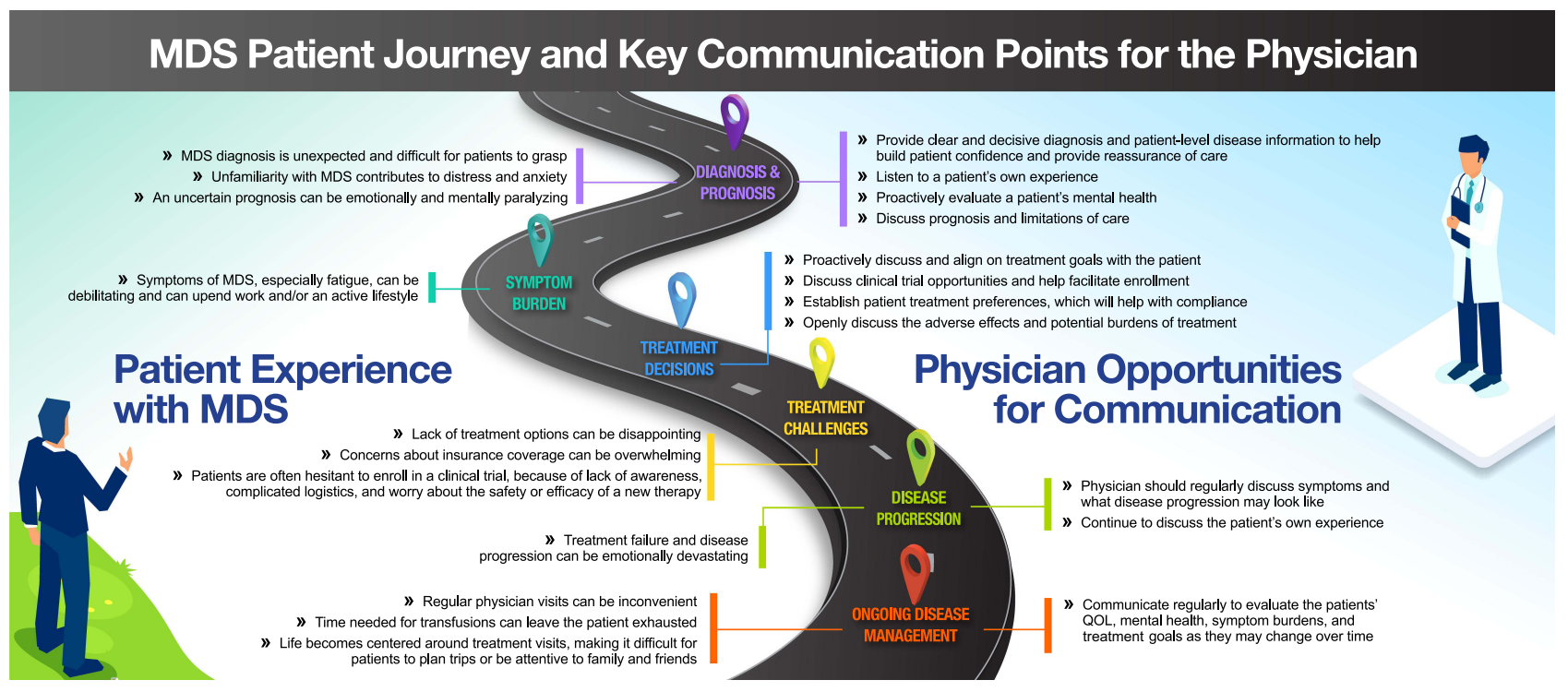

Figure I MDS patient journey and key communication points for the physician. Abbreviations: MDS, myelodysplastic syndrome; QOL, quality of life.

\section{Abbreviations}

COVID-19, coronavirus disease 2019; MDS, myelodysplastic syndrome; QOL, quality of life.

\section{Data Sharing Statement}

The datasets supporting the conclusions of this article are available upon request to the corresponding author.

\section{Ethics Approval and Informed Consent}

Respondents were required to review and sign the consent form to participate in this survey. Participants were informed about the conduct of the survey and that their de-identified data from the survey may be eventually shared with the study sponsor. This survey research was exempt from IRB and ethics approval based on exemptions stated in the HHS Policy for Protection of Human Subjects: 45 CFR Part 46.104 Exempt research - paragraphs (d) (2) (i).

\section{Acknowledgments}

This manuscript is an original work and was prepared according to ICMJE and GPP3 guidelines. All authors have contributed to and approved the manuscript. Editorial support for this manuscript was provided by and Adam Ruth, $\mathrm{PhD}$ of Medical Expressions (Chicago, Illinois) and was funded by Novartis Pharmaceuticals Corporation. All interviewees who provided patient/caregiver perspectives were compensated for a 1-hr interview, paid by Novartis Pharmaceuticals Corporation.

\section{Author Contributions}

All authors made a significant contribution to the work reported, whether that is in the conception, study design, execution, acquisition of data, analysis and interpretation, or in all these areas; took part in drafting, revising or critically reviewing the article; gave final approval of the version to be published; have agreed on the journal to which the article has been submitted; and agree to be accountable for all aspects of the work.

\section{Funding}

Novartis Pharmaceuticals Corporation funded this study. 


\section{Disclosure}

J Soper is a patient with MDS whose perspective is represented in the article and is a member of the MDS Foundation. I Sadek, A Urniasz-Lippel, and D Norton are employees of Novartis Pharmaceuticals Corporation. M Ness is an employee of Inspire. R Mesa has received grants from Celgene (acquired by Bristol Myers Squibb), Incyte, AbbVie, Samus Therapeutics, Inc., Genotech Pharma Pvt. Ltd., Promedior (acquired by Roche), CTI BioPharma Corp., and Constellation Pharmaceuticals, Inc.; and has received consulting fees from Novartis, Sierra Oncology, Inc., La Jolla Pharmaceuticals Company, and Constellation Pharmaceuticals, Inc. The authors report no other conflicts of interest in this work.

\section{References}

1. Troy JD, de Castro CM, Pupa MR, Samsa GP, Abernethy AP, LeBlanc TW. Patient-reported distress in myelodysplastic syndromes and its association with clinical outcomes: a retrospective cohort study. J Natl Compr Canc Netw. 2018;16(3):267-273. doi:10.6004/jnccn.2017.7048

2. Steensma DP, Heptinstall KV, Johnson VM, et al. Common troublesome symptoms and their impact on quality of life in patients with myelodysplastic syndromes (MDS): results of a large internet-based survey. Leuk Res. 2008;32(5):691-698. doi:10.1016/j.leukres.2007.10.015

3. Ria R, Moschetta M, Reale A, et al. Managing myelodysplastic symptoms in elderly patients. Clin Interv Aging. 2009;4:413-423. doi:10.2147/cia. s5203

4. Lown BA, Rosen J, Marttila J. An agenda for improving compassionate care: a survey shows about half of patients say such care is missing. Health Aff (Millwood).2011;30(9):1772-1778. doi:10.1377/hlthaff.2011.0539

5. Street RL Jr, Mazor KM, Arora NK. Assessing patient-centered communication in cancer care: measures for surveillance of communication outcomes. J Oncol Pract. 2016;12(12):1198-1202. doi:10.1200/JOP.2016.013334

6. Steensma DP, Komrokji RS, Stone RM, et al. Disparity in perceptions of disease characteristics, treatment effectiveness, and factors influencing treatment adherence between physicians and patients with myelodysplastic syndromes. Cancer. 2014;120(11):1670-1676. doi:10.1002/cncr.28631

7. Shah J, McKiernan P. Optimizing communication and adherence to iron chelation therapy from diagnosis to treatment in patients with myelodysplastic syndromes. J Adv Pract Oncol. 2016;7(7):707-717.

8. Basch E, Deal AM, Dueck AC, et al. Overall survival results of a trial assessing patient-reported outcomes for symptom monitoring during routine cancer treatment. JAMA. 2017;318(2):197-198. doi:10.1001/jama.2017.7156

9. Caocci G, La Nasa G, Efficace F. Health-related quality of life and symptom assessment in patients with myelodysplastic syndromes. Expert Rev Hematol. 2009;2(1):69-80. doi:10.1586/17474086.2.1.69

10. Epstein RM, Duberstein PR, Fenton JJ, et al. Effect of a patient-centered communication intervention on oncologist-patient communication, quality of life, and health care utilization in advanced cancer: the VOICE randomized clinical trial. JAMA Oncol. 2017;3(1):92-100. doi:10.1001/ jamaoncol.2016.4373

11. Siddon AJ, Hasserjian RP. How I diagnose low-grade myelodysplastic syndromes. Am J Clin Pathol. 2020;154(1):5-14. doi:10.1093/ajcp/aqaa046

12. Garcia-Manero G, Chien KS, Montalban-Bravo G. Myelodysplastic syndromes: 2021 update on diagnosis, risk stratification and management. Am J Hematol. 2020;95(11):1399-1420. doi:10.1002/ajh.25950

13. Ousseine YM, Butow PN, Fenaux P, et al. Association between health literacy, communication and psychological distress among myelodysplastic syndromes patients. Leuk Res. 2018;73:44-50. doi:10.1016/j.leukres.2018.08.020

14. Cook SA, Salmon P, Hayes G, Byrne A, Fisher PL. Predictors of emotional distress a year or more after diagnosis of cancer: a systematic review of the literature. Psychooncology. 2018;27(3):791-801. doi:10.1002/pon.4601

15. Granek L, Nakash O, Ariad S, Shapira S, Ben-David M. Oncologists' identification of mental health distress in cancer patients: strategies and barriers. Eur J Cancer Care (Engl). 2018;27(3):e12835. doi:10.1111/ecc.12835

16. Bailo L, Guiddi P, Vergani L, Marton G, Pravettoni G. The patient perspective: investigating patient empowerment enablers and barriers within the oncological care process. Ecancermedicalscience. 2019;13:912. doi:10.3332/ecancer.2019.912

17. Triplett DP, LeBrett WG, Bryant AK, et al. Effect of palliative care on aggressiveness of end-of-life care among patients with advanced cancer. $J$ Oncol Pract. 2017;13(9):e760-e769. doi:10.1200/JOP.2017.020883

18. Mesa RA, Miller CB, Thyne M, et al. Differences in treatment goals and perception of symptom burden between patients with myeloproliferative neoplasms (MPNs) and hematologists/oncologists in the United States: findings from the MPN Landmark survey. Cancer. 2017;123(3):449-458. doi:10.1002/cncr.30325

19. Lindsley RC. Uncoding the genetic heterogeneity of myelodysplastic syndrome. Hematology Am Soc Hematol Educ Program. 2017;2017(1):447452. doi:10.1182/asheducation-2017.1.447

20. Koutsavlis I. Transfusion thresholds, quality of life, and current approaches in myelodysplastic syndromes. Anemia. 2016;2016:8494738. doi:10.1155/2016/8494738

21. Smith HR. Depression in cancer patients: pathogenesis, implications and treatment (Review). Oncol Lett. 2015;9(4):1509-1514. doi:10.3892/ ol.2015.2944

22. Turner J, Kelly B. Emotional dimensions of chronic disease. West J Med. 2000;172(2):124-128. doi:10.1136/ewjm.172.2.124

23. Haslam A, Herrera-Perez D, Gill J, Prasad V. Patient experience captured by quality-of-life measurement in oncology clinical trials. JAMA Netw Open. 2020;3(3):e200363. doi:10.1001/jamanetworkopen.2020.0363 


\section{Publish your work in this journal}

Patient Related Outcome Measures is an international, peer-reviewed, open access journal focusing on treatment outcomes specifically relevant to patients. All aspects of patient care are addressed within the journal and practitioners from all disciplines are invited to submit their work as well as healthcare researchers and patient support groups. The manuscript management system is completely online and includes a very quick and fair peer-review system. Visit http://www.dovepress.com/testimonials.php to read real quotes from published authors.

Submit your manuscript here: http://www.dovepress.com/patient-related-outcome-measures-journal 